\title{
Characterization of edge turbulence in different states of divertor detachment using reflectometry in the ASDEX Upgrade tokamak
}

\author{
V. Nikolaeva ${ }^{1,2,3}$, L. Guimarais ${ }^{1}$, P. Manz ${ }^{2}$, D. Carralero ${ }^{2}$, M.E. Manso ${ }^{1}$, U. Stroth ${ }^{2,3}$, C. \\ Silva $^{1}$, G.D. Conway ${ }^{2}$, E. Seliunin ${ }^{1}$, J. Vicente ${ }^{1}$, D. Brida ${ }^{2}$, D. Aguiam ${ }^{1}$, J. Santos ${ }^{1}$, A. \\ Silva $^{1}$, ASDEX Upgrade team and MST1 team * \\ ${ }^{1}$ Instituto de Plasmas e Fusão Nuclear, Instituto Superior Técnico, Universidade de Lisboa, \\ Portugal \\ ${ }^{2}$ Max Planck Institute für Plasmaphysik, Boltzmannstr. 2, D-85748 Garching, Germany \\ ${ }^{3}$ Physik-Department E28, Technische Universität München, 85748 Garching, Germany
}

16 February 2018

\begin{abstract}
Transport in the scrape-off layer (SOL) depends on the state of divertor detachment. L-mode discharges were analyzed where the state of divertor detachment is varied through a density rampup. By means of reflectometry measurements at the low (LFS) and the high field side (HFS), midplane density fluctuations are studied for the first time in ASDEX Upgrade simultaneously at both sides of the tokamak. Radial density fluctuation profiles $\left(\delta n_{e} / n_{e}\right)$ increase with radius in both the HFS and the LFS. It is found that in the SOL density fluctuation at the LFS have about a factor of two larger amplitude than at the HFS in agreement with ballooned transport. Density fluctuations at the LFS show a modest variation with increasing background density resulting mainly from a rise of low frequency components. Experimental results are in good agreement with an enhanced convection of filaments at the LFS at the beginning of outer divertor detachment leading to a flatter SOL density profile. In this phase of the discharge, density fluctuations measured at the HFS far-SOL display a strong increase, which may be associated with the presence of faster filaments originated at the LFS.
\end{abstract}

\section{Introduction}

Observations from a large number of tokamak experiments provide clear evidence that the turbulence drive is ballooning-like, favoring the outboard plasma region [1-10]. However, most of the

\footnotetext{
${ }^{*}$ See the author list of Meyer et al, Nucl. Fusion 57102014 (2017)
} 
present knowledge of turbulence comes from measurements at the low-field-side (LFS) scrape-off layer (SOL), while data from the high-field-side (HFS) is scarce and needed because the evaluation of poloidal variations is critical to draw conclusions about turbulent transport [11-13]. Simultaneous fluctuation measurements at the LFS and HFS midplane are therefore essential to characterize edge turbulence and to investigate a possible link between midplane and divertor conditions. In this paper we study density fluctuations in L-mode density ramp discharges where divertor conditions evolve from attached to completely detached. These discharges are characterized by the development of a high-field-side high density (HFSHD) region [14] associated with detachment of the inner divertor that expands up to the midplane [15], leading to strong poloidal asymmetries in the SOL density, which are characterized in detail in Ref. [16]. An enhanced cross-field transport has been reported at the LFS at the beginning of outer divertor detachment leading to a flatter density profile [17-20]. Heretofore, the dependence of turbulence along the detachment has been studied mainly at the LFS, while the HFS may also be influenced by the divertor conditions. Motivated by the relevance of characterizing turbulence at both sides to investigate the possible influence of varying SOL boundary conditions and accompanying enhanced filamentary transport, we use here the unique capabilities of the ASDEX Upgrade (AUG) reflectometry diagnostic able to measure density fluctuations simultaneously at both the LFS and the HFS.

The paper is organized as follows: after the introduction, in section 2 the experiment is described, together with the relevant diagnostics. The methodology used to analyze density fluctuations is briefly described in section 3. The study of turbulence behavior is presented in section 4 . A summary and discussion are given in section 5 .

\section{Experimental setup}

\section{Diagnostics}

Density fluctuations were measured in AUG with a frequency modulated-continuous wave (FMCW) O-mode reflectometry diagnostic operating at fixed frequency. The system is equipped with a homodyne type of detection providing $A(t) \cos (\phi(t))$ signals ( $\phi$ and $A$ being the phase and amplitude, respectively) [21]. This study is based on measurements covering mainly the plasma SOL, at six layers with constant density (one per frequency channel) in K-, Ka- and Q-bands, three at the LFS and three at the HFS, as indicated in Table 1. Each channel uses a single antenna in a mono-static arrangement (the same antenna is used both for emission and reception); antennas are located in-vessel close to the tokamak mid-plane. They feature a small poloidal tilt to favor propagation perpendicular to the magnetic field lines [21,22]. Figure 1 displays the schematic representation of the FM-CW diagnostic in a poloidal section of AUG. The FM-CW system was also operated in swept frequency mode in paired discharges to obtain density profiles at both the HFS and the LFS enabling to localize the layers where fluctuations are measured along the discharge.

Fluctuation data from reflectometry were compared with similar data from a reciprocating probe available at the LFS mid-plane measuring, among other parameters, the ion saturation current 
Table 1: Probing frequencies and respective cut-off densities.

\begin{tabular}{|c|c|c|c|}
\hline Frequency bands (HFS/LFS) & $\mathrm{K}$ & $\mathrm{Ka}$ & $\mathrm{Q}$ \\
\hline $\mathrm{F}[\mathrm{GHz}]$ & 18 & 26 & 36 \\
$n_{e}\left[10^{19} \mathrm{~m}^{-3}\right]$ & 0.40 & 0.84 & 1.61 \\
\hline
\end{tabular}

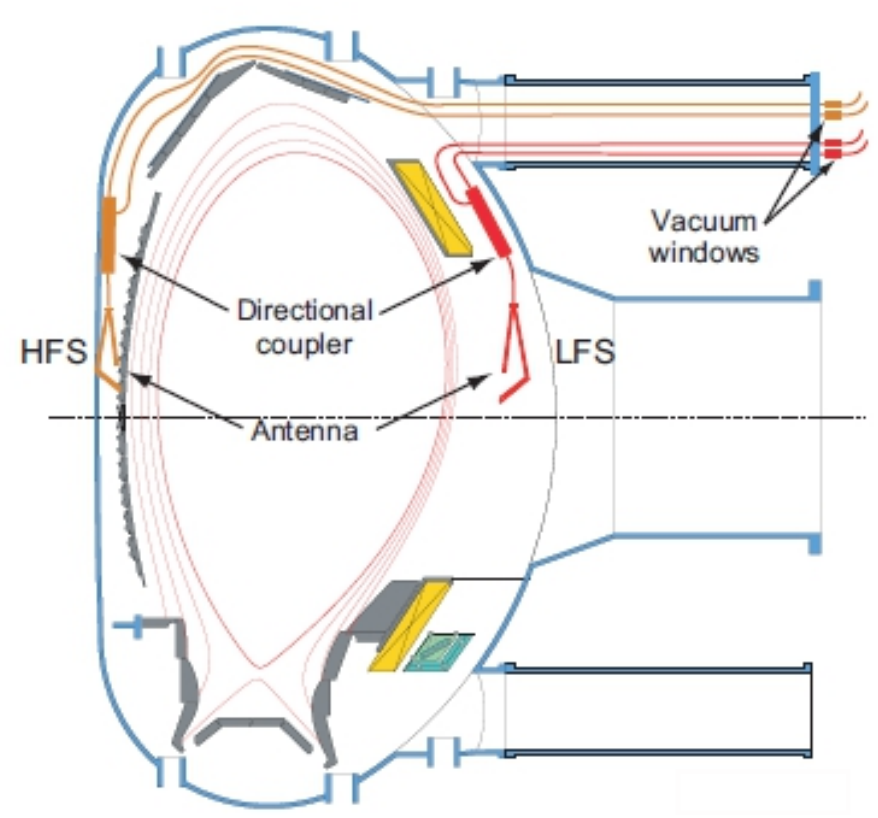

Figure 1: Schematic representation of the FM-CW diagnostic location in a poloidal section of AUG [23].

$\left(I_{s a t} \propto n_{e}\left(T_{e}+T_{i}\right)^{1 / 2}\right)$ at the same acquisition rate as reflectometry of $2 \mathrm{MHz}$ [24].

\section{Description of the experiment}

Density ramp-up discharges were performed in a standard lower single null (LSN) magnetic configuration with the ion $\nabla B$ drift towards the $\mathrm{X}$-point by increasing the deuterium gas injected while maintaining constant levels of additional heating power. The evolution of the plasma parameters measured in the divertor and SOL regions during a typical density ramp-up discharge is shown in Figure 2. A rate of the density ramp-up varies along the discharge, slowing down around 2.7-3.4 s. During the faster ramp in the beginning and the end of the discharge the $d n_{e} / d t$ rate is $\approx 2 \cdot 10^{19} \mathrm{~m}^{-3} / \mathrm{s}$. As the density is continuously ramped up time corresponds roughly to density. Indicated by color are the different divertor states as they have been described in detail in [14]. At low densities the plasma is in the attached state (AS). As the inner divertor detaches the plasma is in the onset state $(\mathrm{OS})[t \approx 2.1-2.4 \mathrm{~s}]$. Then low frequency oscillations occur at the X-point, this is called the fluctuating state (FS) $[t \approx 2.4-3.0 \mathrm{~s}]$. Finally, for $t>3.0 \mathrm{~s}$ the transition to the complete detachment state (CDS) happens when the quasi-coherent radiative X-point fluctuations disappear and also the outer divertor starts to detach from the strike-line regions. The CDS is not 


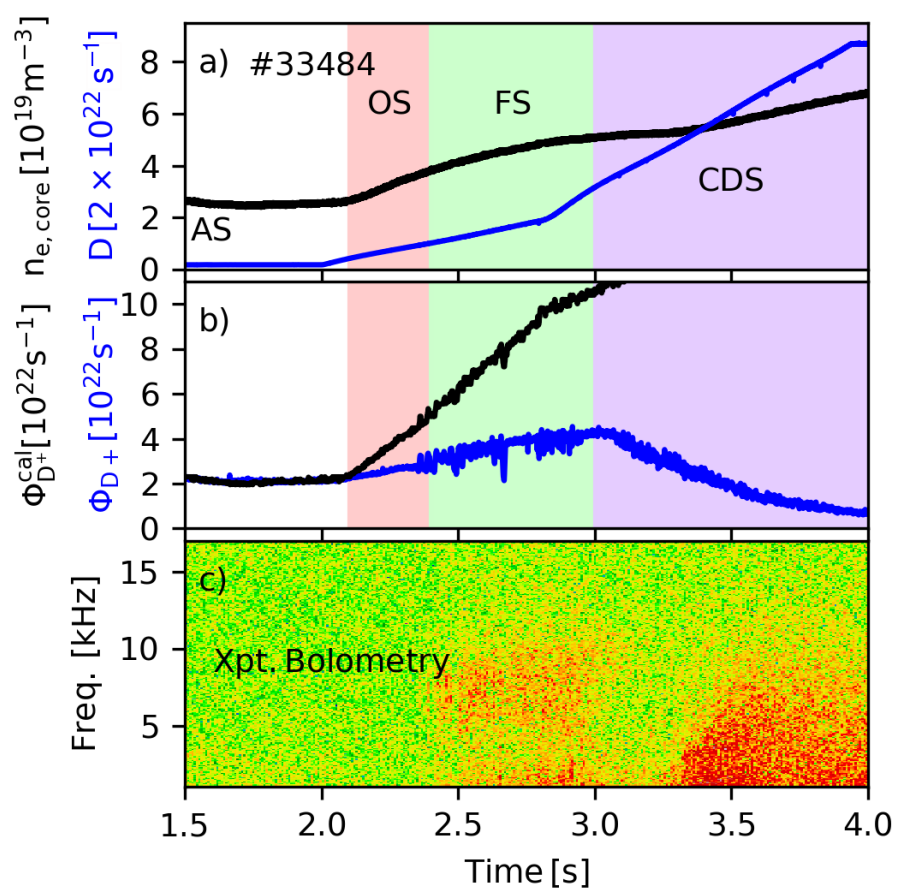

Figure 2: Temporal evolution of the main plasma parameters for discharge \#33484: (a) core lineaveraged density (black), $D_{2}$ fuelling rate (blue); (b) ion flux to the inner divertor measured by the divertor probes $\Phi_{D_{+}}$(blue) and the calculated ion flux assuming a scaling with the square of the line-integrated plasma density $\Phi_{D_{+}}^{c a l}$ (black) [14]; (c) spectrogram of the line-integrated radiated power measured by an AXUV diode (above the X-point). The different detachment states are indicated by the color blocks.

meant to describe the quality of detachment, which is often also described by partially or complete detachment. Density profiles from reflectometry were measured in L-mode paired discharges with constant magnetic parameters: toroidal magnetic field of $B_{t}=-2.5 \mathrm{~T}$ (a negative $B_{t}$ corresponds to the ion $\nabla B$ drift down towards the lower divertor), safety factor of $q_{95} \approx 4$, plasma current of $I_{p}=800 \mathrm{kA}$ and ECRH power of $400 \mathrm{~kW}$. The core and edge line-averaged density are ramped-up during the discharge in a range of $\bar{n}_{e}=2.7-7.2$ and $1.5-4.4 \times 10^{19} \mathrm{~m}^{-3}$, respectively. On discharge \#33484 the FM-CW reflectometer was operated in fixed frequency mode to measure density fluctuations and on discharge \#33483 it was operated in swept mode to obtain density profiles.

As the plasma parameters evolve slowly (typically on the timescale of seconds), to obtain representative density profiles for each divertor state the reflectometry profiles (measured each $1 \mathrm{~ms}$ ) were averaged over $250 \mathrm{~ms}$ ( 250 profiles). The evolution of the radial density profiles along the discharge is displayed in Figure 3. The time intervals where profiles were measured are indicated in the figure caption. The dashed lines indicate the three density layers probed for fluctuation measurements, which move outwards both at the HFS and the LFS as a consequence of the profile evolution. The density profiles provide not only the variable location of the probed layers but also the local density gradients that is needed for the estimation of the density fluctuation amplitudes (see next section 3). 


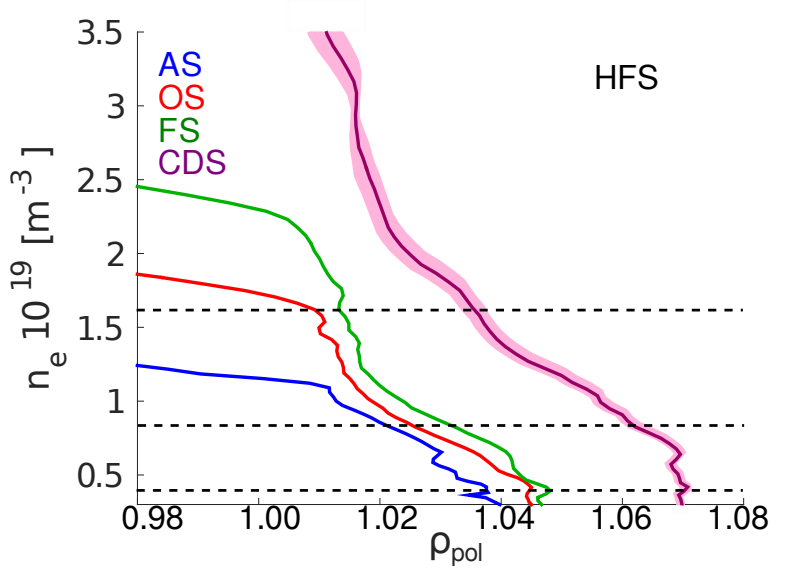

(a) HFS

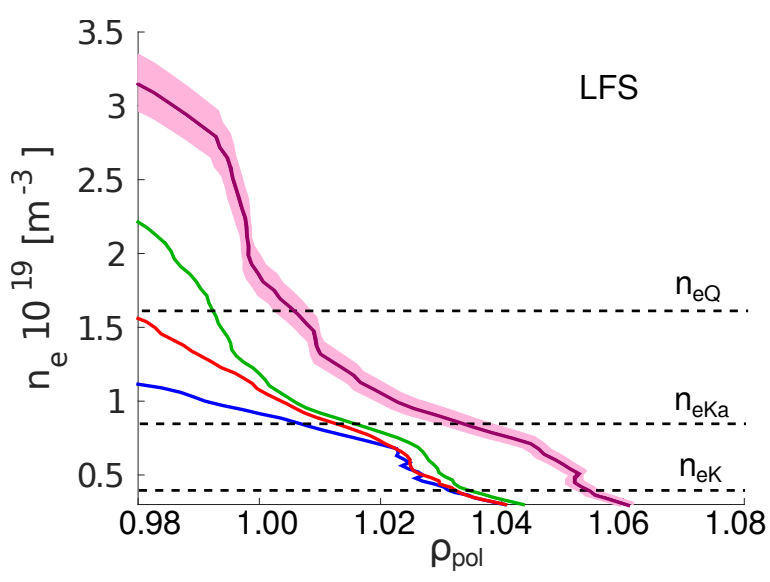

(b) LFS

Figure 3: Density profiles from reflectometry at (a) the HFS and (b) the LFS in different divertor detachment states in discharge \#33483. Profiles are averaged over $250 \mathrm{~ms}$. Time intervals for profile averaging are: (i) AS [1.75-2.0 s]; (ii) OS [2.15-2.4 s]; (iii) FS [2.4-2.65 s], (iv) CDS [3.35-3.6 s]. Probed density layers in K, Ka and Q frequency ranges used in the paired discharge \#33484 are indicated by dashed lines. The shadow regions around the profiles in CDS indicate standard deviation of 250 profiles (corresponding to the selected $250 \mathrm{~ms}$ time ranges).

For low line-average densities corresponding to the initial attached state $(t<2.1 \mathrm{~s})$, the density profiles are roughly HFS/LFS symmetric (Fig. 3). After $t \approx 2.1 \mathrm{~s}$ the inner divertor detaches and the profiles become poloidally asymmetric as a consequence of a strong increase of the SOL density at the HFS. This increase is particularly strong towards the later phase of the discharge as a consequence of the development of the HFSHD region [16]. At the LFS, a pedestal-like structure is formed near the separatrix starting around the fluctuating state, following the increase in the core and edge line-averaged densities (see Figure 3). During complete detachment the LFS density profile broadens with the flat region extending up to $\rho_{\text {pol }} \approx 1.05$ ), as was previously observed by the lithium beam and the reciprocating Langmuir probe diagnostics at AUG [17]. This flattening was shown to occur when a certain divertor collisionality is surpassed leading to an increase of the convective transport caused by filaments [17-20].

\section{Methodology for the density fluctuations estimation from reflec- tometry}

The absolute value of the plasma density fluctuations at selected density layers $n_{e}$ can be estimated from the phase $\phi(t)$ of the O-mode reflectometry signals with probing frequency $F$. The probed density where reflection occurs is derived from the cutoff condition $F=F_{p e}$, where $F_{p e}$ is the electron plasma frequency: $n_{e}=4 \pi^{2} \epsilon_{0} m_{e} F_{p e}^{2} / e^{2}$, where $\epsilon_{0}$ is the vacuum permitivity and $m_{e}$ the electron mass. So the probed density is obtained directly from the incident frequency $F$. The phase response is difficult to interpret because the fluctuations can produce several effects on the probing waves, 
namely scattering, refraction and periodic deformations and/or movements of the reflecting layer. To estimate the fluctuation level $\delta n_{e} / n_{e}, 1 \mathrm{D}$ or $2 \mathrm{D}$ models can be used. For fluctuations where the transverse fluctuation wavelength $\lambda$ is much larger than the probing beam radius $d(\lambda \gg d), 1 \mathrm{D}$ models are usually employed due to their simplicity, such as the Fanack model [25], leading to the estimation of the normalized fluctuation level from the phase as follows,

$$
\frac{\delta n_{e}}{n_{c r}}=\frac{\Delta \phi_{\max }}{\pi \sqrt{2}}\left(\frac{k_{f} / k_{0}}{L_{n} / \lambda_{0}}\right)^{1 / 2}
$$

where $n_{c r}$ is the value of the density at the cut-off, $\Delta \phi_{\max }$ is the maximum phase shift, $k_{f}$ is the wavenumber of the fluctuations, $k_{0}$ is the vacuum wavenumber, $L_{n}$ is the density gradient length at the cutoff layer, and $\lambda_{0}$ is the vacuum wavelength. The 1D C. Fanack model, however, is only valid for small amplitude fluctuations that is not usually the case in the SOL. Furthermore, the model does not cover $2 \mathrm{D}$ and $3 \mathrm{D}$ effects that occur for example when multiple reflections are present. Nevertheless, the validation of 1D model for the absolute density fluctuation was done using fullwave code [26]. A comparison between full-wave simulations and experimental measurements show that results from 1D analysis seems to provide a good estimation of fluctuation levels [27].

As the FM-CW reflectometer provides homodyne signals of $S(t)=A(t) \cos (\phi(t))$ where phase and amplitude are not separated, a method was developed by applying the Hilbert transform, to extract the phase needed to estimate fluctuations [28]. This method was validated by comparison with data from another reflectometry system available at AUG (probing generally the confined region at the LFS) that is equipped with heterodyne detection (providing phase directly) as well as with Langmuir probe data. This new method enables the study of density fluctuations with the FM-CW reflectometer thereby allowing absolute measurements of the density fluctuations at both the HFS and the LFS. However, the phase signal is often characterized by phase jumps, known as phase runaway, that are not related to plasma density fluctuations. Phase runaway has been observed in many tokamaks [29-31] and can be removed by applying a high pass filter. As shown in the next section, in the SOL, the low frequency components (below $15 \mathrm{kHz}$ ) of density fluctuations increase with line-averaged density being dominant at high densities. If a high pass filter with cutoff frequency around $5 \mathrm{kHz}$ is applied to remove phase runaway, the fluctuations levels would be underestimated. Consequently, an alternative method was used when the fluctuation level estimated from the filtered phase is not representative of the broadband fluctuations. The homodyne reflectometry signal can closely display the density fluctuation behaviour (although it cannot provide the absolute level of the density fluctuations), as it was shown experimentally at CTT and DIIID [32]. Therefore, we apply a method that uses the standard deviation of the homodyne signal multiplied by a weighting factor $\sqrt{\lambda_{0} / L_{n}}$ to compensate for the modulated response of reflectometry to different density gradients. The corresponding results are presented in arbitrary units. It will be shown in section 4.2 that the fluctuation properties derived from this method are in good agreement with similar results from the reciprocating Langmuir probe. 


\section{Experimental results}

\subsection{Radial profiles of the density fluctuations at the LFS and the HFS}

An absolute comparison of density fluctuations at the HFS and the LFS can be derived from the phase of the reflected signals (as explained in section 3) at low line-averaged densities or in other cases where the filtering out of the low frequency components does not hamper the analysis of the density fluctuations. Figure 4 displays the radial evolution of density fluctuations at edge and SOL

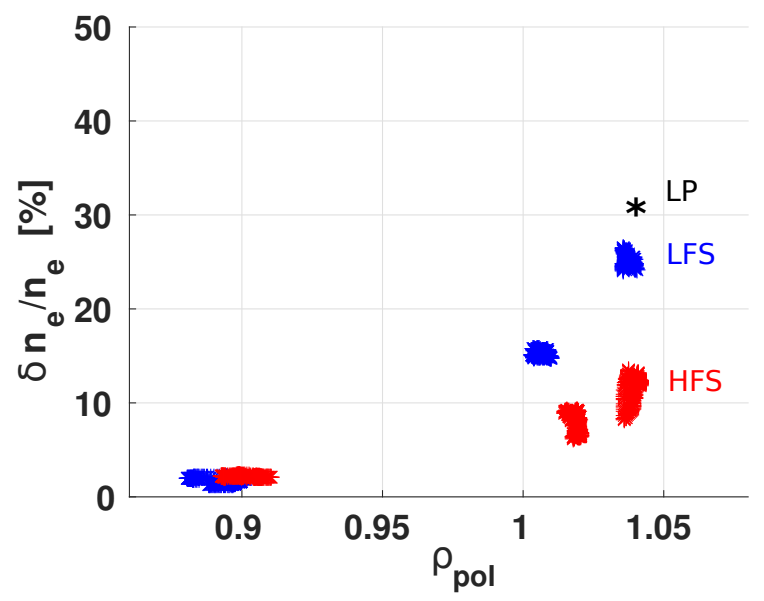

Figure 4: Radial profiles of $\delta n_{e} / n_{e}$ at the LFS and the HFS obtained from FM-CW reflectometry in discharge \#33484 with an edge line-averaged density of $\bar{n}_{e} \approx 1.3 \cdot 10^{19} \mathrm{~m}^{-3}$. The location of the probed layers is measured with the FM-CW system operated in profile mode in the paired discharge \#33483. The star symbols indicate values of $\delta n_{e} / n_{e}$ estimated from the reciprocating Langmuir probe data at the LFS in similar experimental conditions [17].

regions estimated from the FM-CW homodyne phase in discharge \#33484. Data were obtained in the initial part of the discharge when the edge line-averaged density is low $\left(\bar{n}_{e} \leq 1.510^{19} \mathrm{~m}^{-3}\right)$. Also displayed in the figure is the fluctuation amplitude by the reciprocating Langmuir probe inferred from [17] in similar experimental conditions. The density fluctuation amplitudes are seen to increase radially at both the LFS and the HFS as it is typically observed in fusion plasmas $[5,11]$. Levels in the confined region are found to be as low as $\approx 2 \%$ while in the near SOL they range from $15-25 \%$ on the LFS and from $5-15 \%$ on the HFS. The lower values of $\delta n_{e} / n_{e}$ in the HFS SOL than at the LFS (by a factor of $\approx 2$ ) is consistent with ballooned transport. Radial profile of density fluctuations from reflectometry at the LFS matches quite well the Langmuir probe results (taking into account an expected pronounced increase of density fluctuations towards the far SOL [11]) and that density fluctuations by the reciprocating probe were estimated from the ion saturation current measurements ignoring the contribution of temperature fluctuations. This indicates that even in the unfavorable case of high fluctuations at the SOL plasma reflectometry appears to provide a correct estimation of the density fluctuation levels. However, as the validation was performed only for the LFS SOL, we cannot discard differences in the plasma response at low fluctuation levels. Thus, some uncertainties in the ratio of the HFS/LFS fluctuation level cannot be excluded. 


\subsection{Density fluctuations at the LFS SOL}

For the analysis of the density fluctuations along with detachment we consider first the evolution at the LFS and later (in section 4.4) at the HFS. From now on we use the standard deviation of the homodyne reflected signals due to the relevance of the low frequency components of fluctuations, as explained in section 3. Therefore the density fluctuation results are presented in arbitrary units.

The radial profiles of the density fluctuations $\delta n_{e} / n_{e}$ and non-normalized fluctuations $\delta n_{e}$ (obtained multiplying $\delta n_{e} / n_{e}$ by the probing density $n_{e}$ ) in the LFS SOL for discharge \#33484 (characterized in section 2) for each divertor state were estimated from data obtained at three density layers, $n_{e}=0.4,0.84$ and $1.61 \times 10^{19} \mathrm{~m}^{-3}$, using a $42.5 \mathrm{~ms}$ time window (during this time range the line-averaged density varies by $<5 \%$ ). Results are displayed in Figure 5 and Figure 6 , where the color of the points indicates the divertor state. The locations of each probed layer were obtained from the reflectometry density profiles also averaged over $42.5 \mathrm{~ms}$. As the probing densities are fixed the radial coverage follows the profile dynamics.

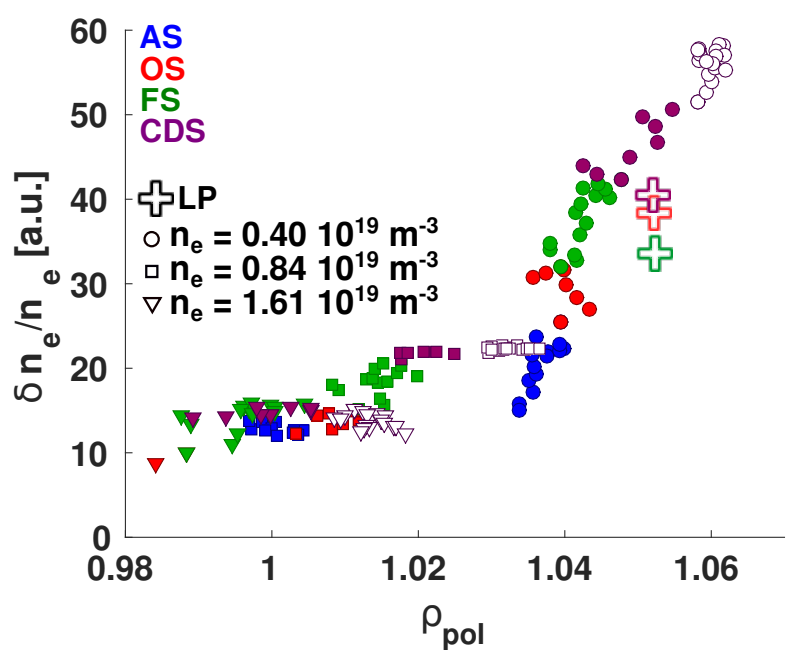

Figure 5: LFS SOL: radial profiles of $\delta n_{e} / n_{e}$ density fluctuations obtained using the standard deviation of the homodyne signal from FM-CW reflectometry in K-, Ka- and Q-bands. Data were measured in discharge \#33484 and were estimated using a $42.5 \mathrm{~ms}$ time window. Color of points corresponds to the different divertor states: attached (blue), onset (red), fluctuating (green) and complete detachment state (purple, closed symbols attributed to the beginning of the state from 3 to $3.35 \mathrm{~s}$, open circles to the later part of the state). Symbols correspond to different probed densities: $n_{e K}=0.40 \cdot 10^{19} \mathrm{~m}^{3}$ (circles), $n_{e K a}=0.84 \cdot 10^{19} \mathrm{~m}^{3}$ (squares) and $n_{e Q}=1.61 \cdot 10^{19} \mathrm{~m}^{3}$ (triangles). Cross symbols correspond to the density fluctuations estimated using the ion saturation current $I_{\text {sat }}$ signal measured by the reciprocating Langmuir probe in the similar discharge \#30303.

Relative density fluctuations $\delta n_{e} / n_{e}$ are seen to increase with radius for all the divertor states (Figure 5). Changes with the density along the discharge seem to depend on $\rho_{\text {pol }}$ locations. Close to the separatrix $\left(\rho_{p o l} \approx 1.00\right)$, density fluctuations do not display significant changes with the divertor state, while at $\rho_{\text {pol }} \approx 1.04$ density fluctuations increase along the evolution of divertor from the attached towards the complete detachment state. In the completely detached state, the radial 


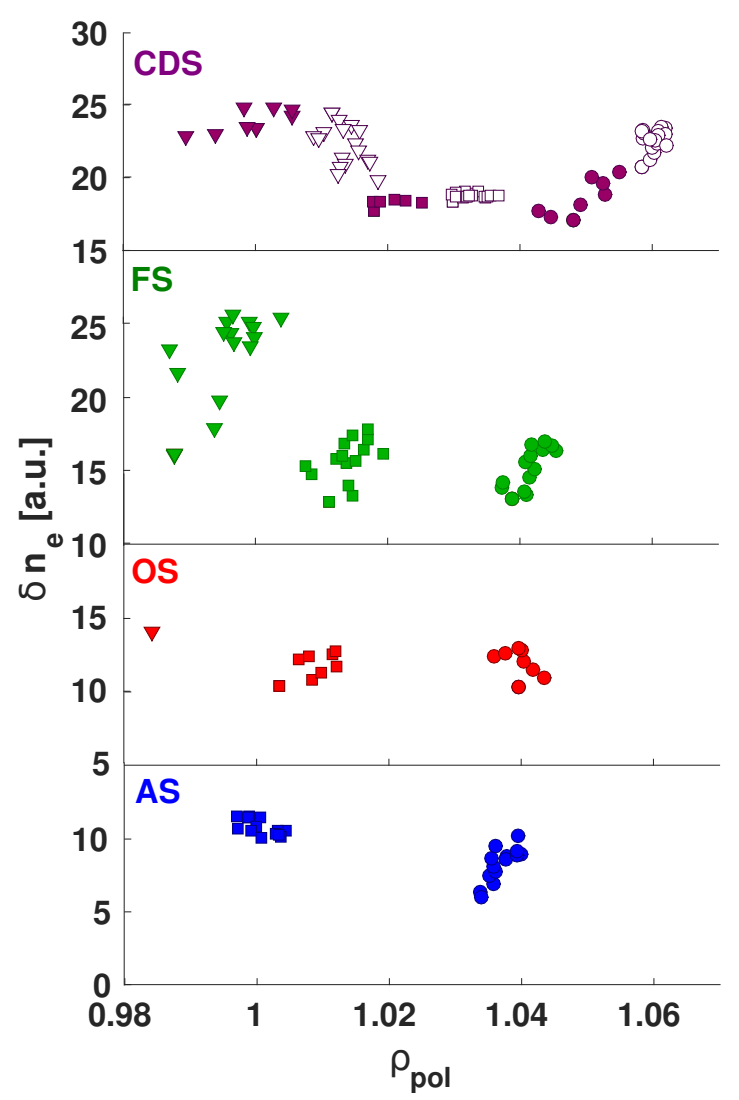

Figure 6: LFS SOL: radial profiles of non-normalized $\delta n_{e}$ density fluctuations obtained by multiplying $\delta n_{e} / n_{e}$ (Figure 5) to the probing densities. Symbols correspond to different probed densities: $n_{e K}=0.40 \cdot 10^{19} \mathrm{~m}^{3}$ (circles), $n_{e K a}=0.84 \cdot 10^{19} \mathrm{~m}^{3}$ (squares) and $n_{e Q}=1.61 \cdot 10^{19} \mathrm{~m}^{3}$ (triangles). In the complete detachment state (the top plot), closed symbols are attributed to the beginning of the state from 3 to $3.35 \mathrm{~s}$ and open circles to the later part of the state.

coverage is extended towards the far SOL $\left(\rho_{\text {pol }} \approx 1.06\right)$ following the density profile outward shift (see Figure 3), which hampers the comparison with fluctuations in the other detachment states. For the comparison with other detachment states, we use here density fluctuation levels of the ion saturation current signal from the reciprocating Langmuir probe for a similar density ramp discharge (\#30303), measured at $\rho_{\text {pol }} \approx 1.05-1.055$ in three successive plunges during the onset, the fluctuating and the complete detachment state. At this location the dependence of the $I_{\text {sat }}$ fluctuation level on the density is modest, with a lower value seen in the fluctuating state. Our results suggest therefore that density fluctuation levels have a modest dependence on the line-averaged density in the near and far SOL regions, contrary to the observation around $\rho_{\text {pol }} \approx 1.04$ where density fluctuations increase along with the density ramp. Non-normalized density fluctuations $\delta n_{e}$ depicted in Figure 6 (obtained by multiplying the data in Figure 5 by the probing density value) are seen to decrease with radius in the near SOL that can be associated with parallel transport losses. In the later phase of the complete detachment state $\delta n_{e}$ display an increase in the far SOL, which can not be attributed to the change of the characteristics of the filaments. However, it may be associated with other sources of density perturbations for instance resulting from fast variations in the divertor 
condition. Finally, it cannot be excluded that at such high level of fluctuations in the far SOL, 2D and $3 \mathrm{D}$ propagation effects might play a role making the interpretation of the reflectometry signals more complicated.

\subsection{Frequency-resolved analysis of density fluctuations}

A first picture of the typical density fluctuations at each divertor state can be inferred from the time

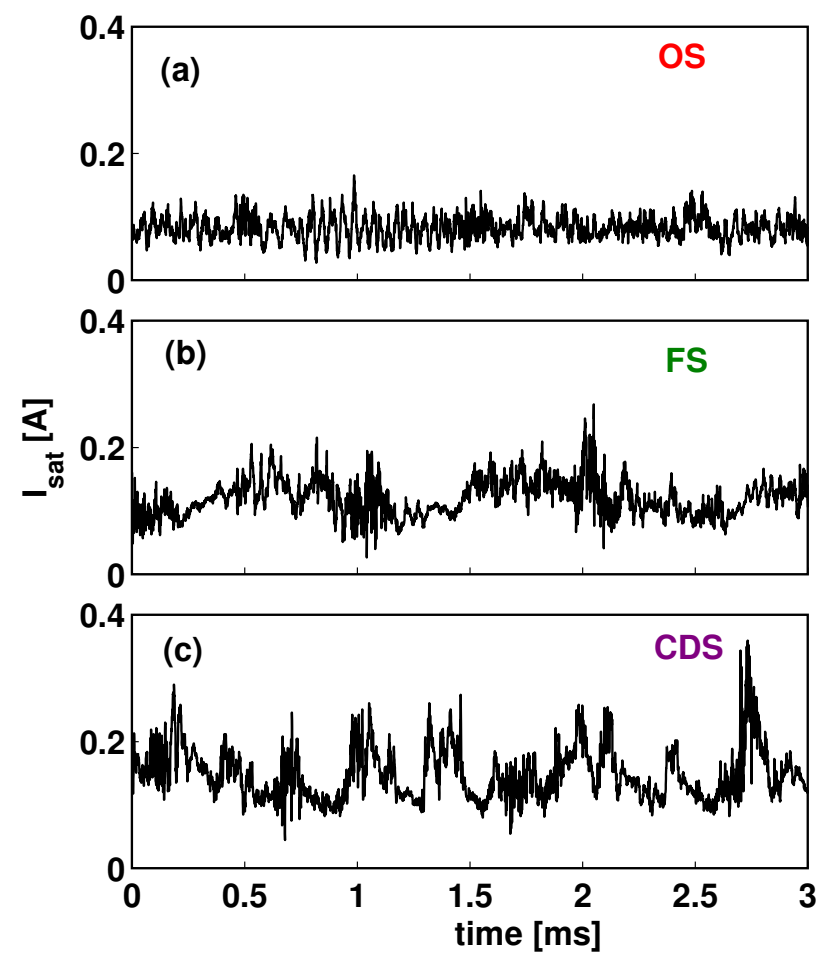

Figure 7: Ion saturation current time traces obtained by the reciprocating Langmuir probe for $\left[\rho_{\text {pol }}: 1.050-1.054\right]$ in $3 \mathrm{~ms}$ periods during 3 plunges corresponding to the (a) onset, (b) fluctuating and (c) complete detachment states in discharge \#30303.

traces of $I_{\text {sat }}$ obtained by the reciprocating Langmuir probe in three successive plunges in discharge \#30303 as displayed in Figure 7. At the onset state, fluctuations are characterized by fast, low amplitude fluctuations while during complete detachment large $I_{\text {sat }}$ spikes are seen that are associated with filaments, which become significantly larger and less frequent. In the fluctuating state the amplitude of the filaments increases with respect to the onset state and pronounced low frequency oscillations are superimposed possibly related to the oscillations in the divertor conditions [14]. The increase of filamentary activity along the discharge can be quantified by the skewness of the fluctuations for the different divertor states: the values are about $0.3,0.5$ and 1.1 in the onset, fluctuating and complete detachment state, respectively.

To characterize the dominant frequency components of the midplane fluctuations along the divertor detachment, we present the evolution of the fluctuation power spectra from reflectometry probing the far SOL layer at the LFS $\left[\rho_{\text {pol }}: 1.04-1.06\right]$, as shown in Figure 8 (left). For comparison, 

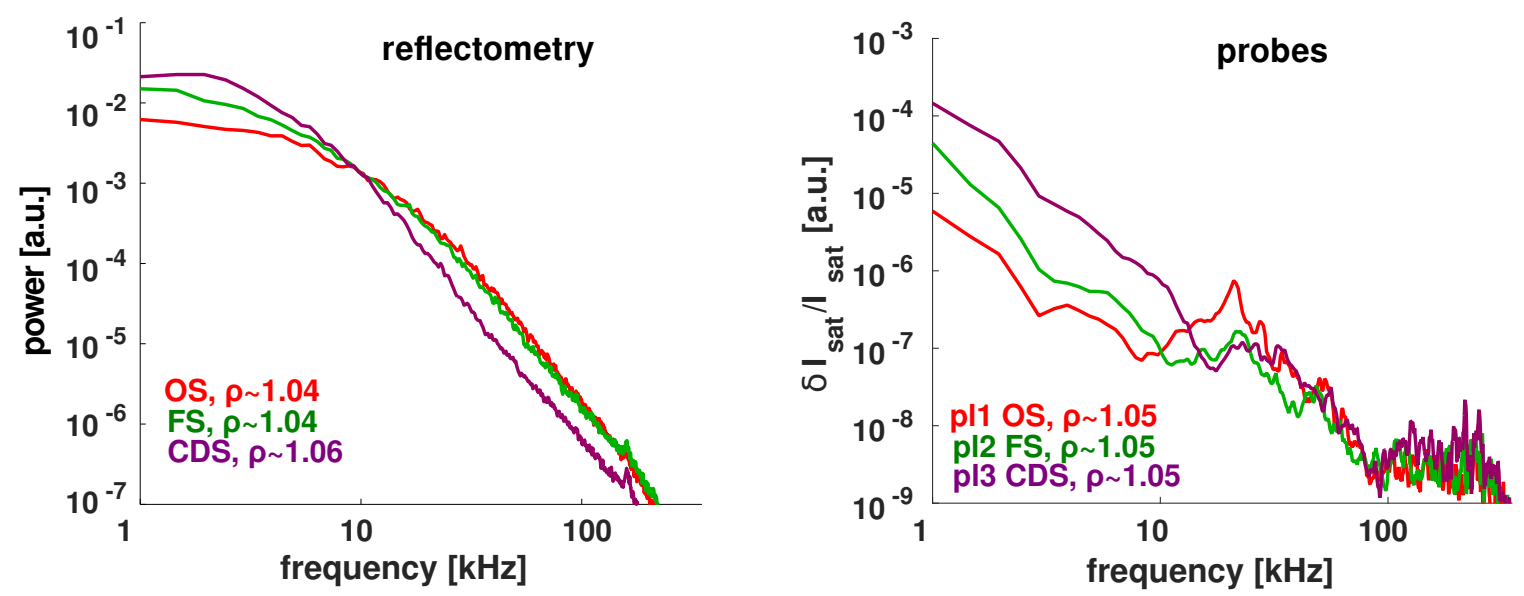

Figure 8: At the left power spectra of the K-band LFS homodyne signal measured by the FM-CW reflectometer in the SOL $\left[\rho_{p o l}=1.04-1.06\right]$ in onset, fluctuating and complete detachment states during discharge \#33484; at the right power spectra of the ion saturation current measured by the reciprocating Langmuir probe at the mid-plane LFS SOL in the similar discharge \#30303 during the same detachment states.

Figure 8 at the right depicts the power spectra from the $I_{\text {sat }}$ signal measured by the reciprocating Langmuir probe at $\rho_{\text {pol }} \approx 1.05$. Fluctuations with a broad frequency spectrum are obtained from both diagnostics showing that the increase of the fluctuation amplitude described above (see Figure 5) results mainly from an increase of the low frequency components $(f<15 \mathrm{kHz})$.

As indicated by the time traces of the ion saturation current from the reciprocating probe (see figure 7), the increase of low frequency components in the fluctuating state is associated with both filaments and low frequency oscillations at the midplane, while in the complete detachment state the increase should result mainly from the increase in the filamentary activity. Indeed, during the complete detachment state fluctuations are dominated by large, infrequent filaments lasting more than $0.1 \mathrm{~ms}$ (see figure 7) in good agreement with the frequency range where the power spectrum is observed to increase. We can therefore conclude that the increase in the low frequency fluctuations measured by reflectometry at the LFS SOL is due to an increase of filamentary activity. The decrease of the high frequency $(f>15 \mathrm{kHz})$ components seen during the CDS in the power spectra from reflectometry can so far not be explained requiring further investigation with better spatial resolution.

\subsection{Density fluctuations at the HFS SOL}

We turn now our attention to the behavior of the fluctuations at the HFS. HFS/LFS asymmetries are expected as a consequence of ballooned transport in the SOL. The link between the LFS and the HFS SOL and the possible influence of the divertor conditions will also be investigated.

Figure 9 presents the evolution of the relative density fluctuations at the HFS SOL in discharge \#33484 derived from reflectometry signals probing the density layers (a) $n_{e K}=0.40 \cdot 10^{19} \mathrm{~m}^{3}(\mathrm{~K}$ band) and (b) $n_{e K a}=0.84 \cdot 10^{19} \mathrm{~m}^{3}$ (Ka-band). The locations of the two probed layers are displayed 

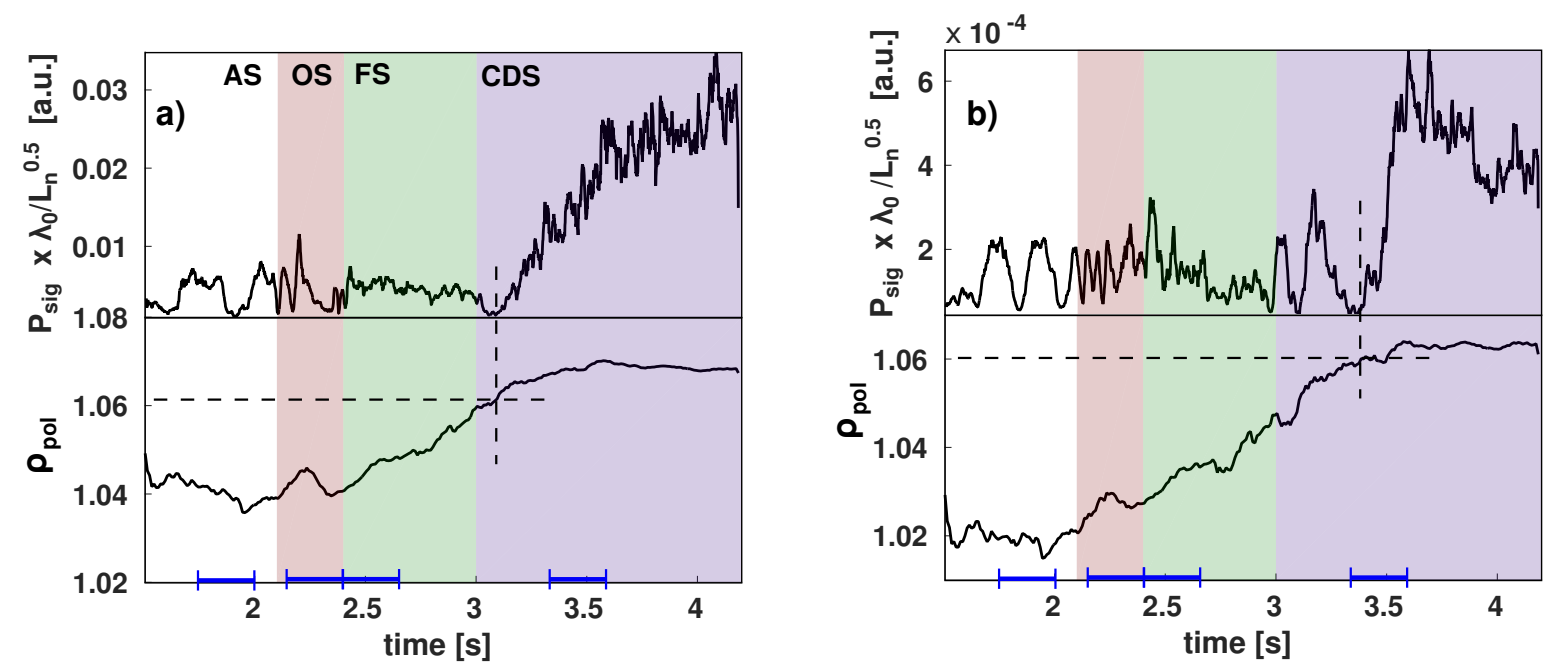

Figure 9: Time traces of the standard deviation of the FM-CW reflectometer signals from (a) the K- and (b) the Ka-band at the HFS SOL in discharge \#33484 multiplied by $\sqrt{\lambda_{0} / L_{n}}$ inferred from the paired discharge \#33483. The variable location of the probed density layers $n_{e K}$ and $n_{e K a}$ is shown at the bottom of each plot. The typical error of $\rho_{\text {pol }}$ is $\lesssim 0.005$. Divertor detachment states (AS, OS, FS and CDS) are indicated. The time intervals $\Delta t=250 \mathrm{~ms}$ shown in blue indicate the periods used to estimate the average properties of density profiles and fluctuations in each detachment state.

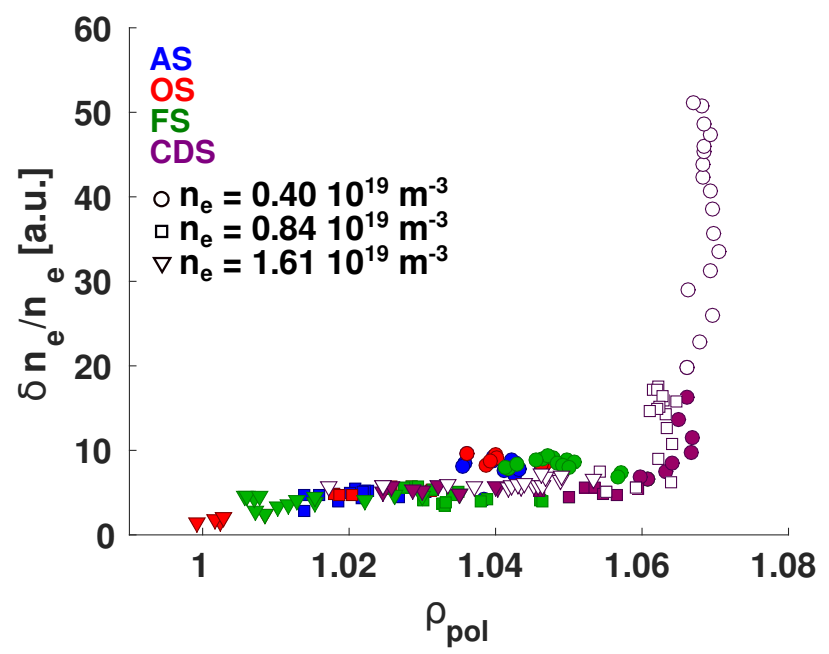

Figure 10: HFS SOL: radial profiles of relative $\delta n_{e} / n_{e}$ density fluctuations obtained using the standard deviation of the homodyne signal from FM-CW reflectometry in K-, Ka- and Q-bands. Data were measured in discharge \#33484 and were estimated using a $42.5 \mathrm{~ms}$ time window. Color of points corresponds to the different divertor states: attached (blue), onset (red), fluctuating (green) and complete detachment state (purple, closed symbols attributed to the beginning of the state from 3 to $3.35 \mathrm{~s}$, open circles to the later part of the state). Symbols correspond to different probed densities: $n_{e K}=0.40 \cdot 10^{19} \mathrm{~m}^{3}$ (circles), $n_{e K a}=0.84 \cdot 10^{19} \mathrm{~m}^{3}$ (squares) and $n_{e Q}=1.61 \cdot 10^{19} \mathrm{~m}^{3}$ (triangles). 
in the two lower graphs. As illustrated in Figure 9, there is no clear trend of density fluctuations behaviour at the HFS SOL until the complete detachment state, when the most pronounced changes are observed. In particular, fluctuations at the HFS do not increase in the fluctuating state contrary to the LFS, where an enhancement of filamentary transport and low frequency oscillations were observed (Figs. 5 and 7 ).

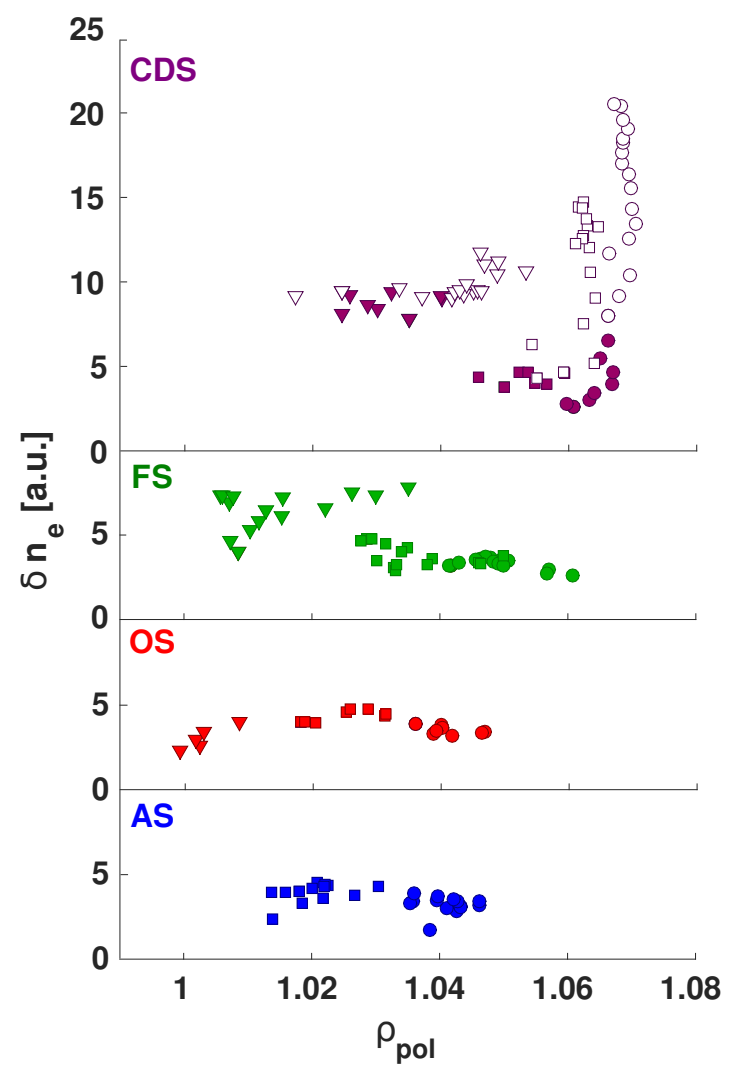

Figure 11: HFS SOL: radial profiles of non-normalized $\delta n_{e}$ density fluctuations obtained by multiplying $\delta n_{e} / n_{e}$ (Figure 10) to the probing densities. Symbols correspond to different probed densities: $n_{e K}=0.40 \cdot 10^{19} \mathrm{~m}^{3}$ (circles), $n_{e K a}=0.84 \cdot 10^{19} \mathrm{~m}^{3}$ (squares) and $n_{e Q}=1.61 \cdot 10^{19} \mathrm{~m}^{3}$ (triangles). In the complete detachment state (the top plot), closed symbols are attributed to the beginning of the state from 3 to $3.35 \mathrm{~s}$ and open circles to the later part of the state.

However, during complete detachment the situation changes, the relative density fluctuations on the HFS start to increase significantly when the probed layer reaches a certain radial location in the far SOL $\left(\rho_{p o l} \approx 1.06\right)$. This justifies why the increase of fluctuations occurs first at the K-band $n_{e K}$ (for $t \approx 3.05 \mathrm{~s}$ ) and later at the Ka-band signal $n_{e K a}$ (for $t \approx 3.40 \mathrm{~s}$ ). The radial profiles of the density fluctuations for the different divertor states shown in Figure 10 (obtained with a $42.5 \mathrm{~ms}$ time window from K-, Ka- and Q-band data) confirm the modest evolution of the HFS fluctuations until complete detachment is reached and a weak radial variation up to $\rho_{\text {pol }} \approx 1.06$. During the complete detachment state, density fluctuation profiles exhibit a sharp increase in the far SOL $\left(\rho_{p o l} \approx 1.06\right)$. However, as reflectometry probes this region only when the density there is high enough to reflect the probing waves, we cannot exclude the possibility that fluctuations in the 
far SOL region may rise before the complete detachment state.

It was previously shown [17] that during complete detachment the radial velocity of the filaments increases, leading to broad density profiles at the LFS. As intermittent radial transport is poloidally asymmetric favoring the LFS [1-10], filaments will propagate along the field lines and they are expected to reach the far SOL at the HFS. Assuming a typical radial velocity of $300 \mathrm{~m} / \mathrm{s}$ characteristic of the complete detachment state [17] and propagation at sound speed along the field lines, filaments should be detected in the HFS far SOL around $\rho_{\text {pol }} \approx 1.1$. This is further out than observed but still in reasonable agreement with experimental findings from reflectometry, taking into account that the radial velocity of filaments coming from the LFS midplane may not be constant radially across the SOL and also they can be originated at the top of the LFS resulting in shorter paths to the HFS midplane. The fact that non-normalized density fluctuations depicted in Figure 11 increase in the far-SOL gives further evidence that these fluctuations are not due to local radial transport but rather to parallel transport originated at the LFS. Therefore, it may be concluded that under certain divertor conditions, such as occurring during the complete detachment state, the HFS SOL can be strongly influenced by the presence of the turbulent structures coming from the LFS.

\section{Summary}

Experimental studies performed at AUG with reflectometry at the high field side (HFS) and the low field side (LFS) in L-mode discharges where the divertor states varied through a density rampup, show that density fluctuations at the midplane are sensitive to the divertor conditions. Radial relative density fluctuation profiles $\left(\delta n_{e} / n_{e}\right)$ increase with radius at both the HFS and the LFS. It is found that in the SOL density fluctuations at the LFS have about a factor of two larger amplitudes than at the HFS in agreement with ballooned transport.

Results from reflectometry reveal that density fluctuation levels on the LFS have a modest dependence on the line-averaged density close to the separatrix at $\rho_{p o l}=1.00-1.01$ and also around $\rho_{p o l}=1.05$ as seen from the reciprocating Langmuir probe data. This is in contrast to the observations around $\rho_{\text {pol }}=1.04$ where density fluctuations from reflectometry increase along with the density ramp. The increase of density fluctuation levels in the complete detachment state results mainly from an increase of the low frequency components $(<15 \mathrm{kHz})$ also observed in the reciprocating Langmuir probe data that is in agreement with the generally accepted view that radial transport at the LFS SOL is dominated by the filamentary activity. As the plasma density is ramped-up, less frequent and faster filaments are observed when a density shoulder is formed on the LFS [17].

When filaments become faster and less frequent at the LFS, relative density fluctuations at the HFS strongly increase in the far SOL region $\left(\rho_{p o l}>1.06\right)$, which can be interpreted as a presence of faster filaments that propagate parallel to the magnetic field lines from the LFS to the HFS. Although the present spatial coverage of FM-CW reflectometry at high density is limited, 
results indicate that under certain divertor conditions, such as those occurring in the complete detachment state, the HFS SOL may be strongly influenced by the presence of turbulent structures originated on the LFS. However, due to lack of data we cannot conclude if some of the changes, for example the decrease of the high frequency components of the density fluctuations during complete detachment, can be attributed to the radial variations of the probing layers or to the evolution of the fluctuations. This requires further investigation that would benefit from an increased spatial coverage using additional probing frequencies in an upgraded FM-CW diagnostic.

This work presents for the first time at AUG simultaneous measurements of the density fluctuations at the HFS and the LFS that provide a consistent picture of the poloidal asymmetry in the density fluctuations as well as of the influence of the divertor condition on the density fluctuations. The capability of FM-CW reflectometry to measure simultaneously both at the LFS and the HFS opens the way to address key topics such as the link between the inboard and outboard plasma regions. This can contribute towards achieving a global picture of the SOL transport characteristics in fusion devices.

\section{Acknowledgement}

This work was carried out within the framework of the Erasmus Mundus International Doctoral College in Fusion Science and Engineering (FUSION-DC) and the EUROfusion Consortium. It has received funding from the Euratom research and training programme 2014-2018 under grant agreement No633053. IST activities also received financial support from "Fundação para a Ciência

e Tecnologia" through project UID/FIS/50010/2013. The views and opinions expressed herein do not necessarily reflect those of the European Commission.

\section{References}

[1] M. Endler et al., Nucl. Fusion 35, 1307 (1995)

[2] B. LaBombard and B. Lipschultz, Nucl. Fusion 27, 81 (1987)

[3] V.A. Vershkov et al., J. Nucl. Mater. 241243, 873 (1997)

[4] J.L. Terry et al., Phys. Plasmas 10, 1739 (2003)

[5] N. Smick et al., Journal of Nuclear Materials 337339, 281285 (2005)

[6] C. Silva et al., Plasma Phys. Control. Fusion 53, 085021 (2011)

[7] G.S. Kirnev et al., Nucl. Fusion 45, 459 (2005)

[8] R. Pitts, G. Vayakis and G.F. Matthews, J. Nucl. Mater. 175177, 893 (1990)

[9] J. Stockel et al., Plasma Phys. Control. Fusion 47, 635 (2005) 
[10] G.R. Tynan et al., Phys. Rev. Lett. 68, 3032 (1992)

[11] S.J. Zweben, et al., Plasma Phys. Control. Fusion 49, S1S23 (2007)

[12] M. Endler et al., Plasma Phys. Control. Fusion 41, 1431 (1999)

[13] B. LaBombard et al., Nucl. Fusion 44, 1047-1066 (2004)

[14] S. Potzel et al., Nucl. Fusion 54, 013001 (2014)

[15] S. Potzel et al., J. Nucl. Mater. 462, 541 (2015)

[16] L. Guimarais et al., Nucl. Fusion 58, 026005 (2018)

[17] D. Carralero et al., Nucl. Fusion 54, 123005 (2014)

[18] D. Carralero et al., Phys. Rev. Lett. 115, 215002 (2015)

[19] D. Carralero et al., Nucl. Fusion 57, 056044 (2017)

[20] J.A. Boedo J. Nucl. Mater. 390391, 2937 (2009)

[21] A. Silva et al., Rev. Sci. Instrum., 67(4138), 4125 (1996)

[22] S. Graça et al., 40th EPS Conf., Espoo, Finland (2013)

[23] A. Silva PhD Thesis 'The ASDEX Upgrade broadband microwave reflectometry system', Instituto Superior Tcnico (2006)

[24] B. Nold, G. D. Conway, T. Happel, et al., Plasma Phys. Control. Fusion 52, 065005 (2010)

[25] C. Fanack et al., Plasma Phys. Control. Fusion 38, 1915 - 1930 (1996)

[26] A. Casati et al., Phys. Rev. Lett. 102, 165005 (2009)

[27] T. Gerbaud, F. Clairet, R. Sabot, and A. Sirinelli, Rev. Sci. Instrum., 77, 10E928 (2006)

[28] V. Nikolaeva, M.E. Manso, C. Silva, G.D. Conway, L. Guimarais, A. Silva, J. Vicente, J. Santos, U. Stroth and the ASDEX Upgrade team, Methodology for estimation of the density fluctuation level at the LFS and the HFS from homodyne reflectometry, Rev. Sci. Instrum. in preparation

[29] E. Mazzucato et al., Phys. Rev. Lett. 7ry(15), 3145 (1996)

[30] B. Brânas et al., Rev. Sci. Instrum., 70(1), 1025 (1999)

[31] K. Shinohara et al., Fusion Eng. Des., 34-35, 433 (1997)

[32] T.L. Rhodes et al., Plasma Phys. Control. Fusion 40, 493-510 (1998) 